between the new study and the previous one, including our study's focus on the construct of health loss rather than welfare loss, and the previous study's reliance on a small panel of health-care professionals and lack of direct comparison between outcomes. We must avoid the trap of treating previous weights as if they were a reference standard. The updated study had among its aims to address limitations in previous measurement work, and we have made every effort to ensure that it does so with an empirically rich, scientifically valid approach.

Finally, it is useful to compare the new disability weights with results from other empirical studies that have estimated healthstate weights for vision loss and blindness. Systematic review is needed here. In the meantime, we find relevant comparisons in studies of patients with diabetes that have used multivariable methods to control for confounding factors and comorbidities. These studies have reported weights for blindness that are similar to $^{3}$ or significantly lower than ${ }^{4}$ ours.

The lay descriptions for vision loss and blindness in our study were developed collaboratively, with extensive consultation, revision, and finally agreement from the Vision Loss Expert Group. We look forward to continuing to engage with the group to advance research on this topic through careful empirical inquiry.

We declare that we have no conflicts of interest.

Joshua A Salomon, Theo Vos, *Christopher J L Murray

cjlm@u.washington.edu

School of Public Health, Harvard University, Boston, MA, USA (JAS); School of Population Health,

University of Queensland, Brisbane, QLD, Australia (TV); and Institute for Health Metrics \& Evaluation, University of Washington in Seattle, Seattle, WA 98195, USA (CJLM)

1 Salomon J, Vos T, Hogan D, et al. Common values in assessing health outcomes from disease and injury: disability weights measurement study for the Global Burden of Disease Study 2010. Lancet 2012; 380: 2129-43.
2 Murray CJL. Rethinking DALYs. In: Murray CJL, Lopez AD, eds. The global burden of disease: a comprehensive assessment of mortality and disability from diseases, injuries and risk factors in 1990 and projected to 2020. Cambridge, MA: Harvard School of Public Health, 1996: 1-98.

3 Coffey JT, Brandle M, Zhou H, et al. Valuing health-related quality of life in diabetes. Diabetes Care 2002; 25: 2238-43.

4 Clarke PM, Simon J, Cull CA, Holman RR. Assessing the impact of visual acuity on quality of life in individuals with type 2 diabetes using the short form-36. Diabetes Care 2006; 29: 1506-11.

\section{Reduction in child mortality in Niger}

Agbessi Amouzou and colleagues' explanation of Niger's impressive reduction in under-5 mortality (Sept 29, p 1169) ${ }^{1}$ shows how healthsector interventions can dramatically improve child survival and reduce health inequities. It also supports calls to prioritise the most deprived communities.2 However, the study raises the question of how to continue this progress. One in eight children in Niger is still likely to die before the age of 5 years, and neonatal survival has improved little.

If Niger, and other countries with high levels of child deaths, are to continue reductions in under-5 mortality (and even realise aspirations to reduce it to no more than 20 per 1000 livebirths by $2035^{3}$ ), there will be diminishing returns if improvements are only made in the delivery of health services. For example, if the proportion of births to women with secondary education or higher remains at less than $5 \%$ in Niger, these further goals are unlikely to be achieved. To sustain the gains seen in Niger, continued efforts will be needed not only to expand interventions to pregnant women and neonates, but also to collaborate with those beyond the health sector to address social determinants.

Resolute efforts by the Government and communities of Niger have been aided by global partners to improve the delivery of health services. ${ }^{4}$ Yet these combined efforts seem to have had much less effect on reducing poverty or increasing maternal education, which are key contributors to ensuring child survival and development, with the latter especially important in the neonatal period. ${ }^{5}$ The global community needs to conceive efforts to improve social determinants as an integral part of efforts to reduce child deaths, at the same time as helping to replicate the improvements in health service delivery that Niger shows is possible even in challenging contexts.

All authors are staff members of UNICEF, which provided support for the original study. However, we were not involved with the original paper. These are the authors' views and do not necessarily reflect the views or policies of UNICEF.

*Kumanan Rasanathan, Theresa Diaz, Julia C Kim, Mark Young

krasanathan@unicef.org

Health Section, UNICEF, New York, NY 10017, USA

1 Amouzou A, Habi O, Bensaïd K, for the Niger Countdown Case Study Working Group. Reduction in child mortality in Niger: a Countdown to 2015 country case study. Lancet 2012; 380: 1169-78.

2 Lake A. A tipping point for child survival, health and nutrition. Lancet 2012; 380: 1286-87.

3 Glass RI, Guttmacher AE, Black RE. Ending preventable child death in a generation. JAMA 2012; 308: 141-42.

4 Sanda S. Niger's success in child survival. Lancet 2012; 380: 1127-28.

5 Gakidou E, Cowling K, Lozano R, Murray C). Increased educational attainment and its effect on child mortality in 175 countries between 1970 and 2009: a systematic analysis. Lancet 2010; 376: 959-74.

The Article by Agbessi Amouzou and colleagues $^{1}$ is a welcome effort to document the successful reduction in the under- 5 mortality rate (U5MR) in Niger. Amouzou and colleagues suggest that universal access to primary health care, mass campaigns, and nutrition programmes are the main strategies responsible for these changes. However, there remain some unanswered questions which would benefit from a more in-depth analysis to explain the drivers of changes in child mortality in this country.

As reported by Amouzou and colleagues, health posts and community health workers (CHWs) seem to be pivotal in increasing geographical 


\begin{tabular}{|lc|}
\hline & Contribution to reduced U5MR \\
\hline Universal access, including health posts & $22 \%$ \\
Care seeking for malaria & $9 \%$ \\
Care seeking for pneumonia & $8 \%$ \\
Use of ORS and zinc & $5 \%$ \\
Mass campaigns/outreach services & $45 \%$ \\
Hib vaccination & $4 \%$ \\
ITN ownership & $25 \%$ \\
Measles vaccination & $5 \%$ \\
Tetanus vaccination in pregnancy & $2 \%$ \\
Vitamin A supplementation & $9 \%$ \\
Programmes for nutrition & $19 \%$ \\
Reduction in wasting & $9 \%$ \\
Reduction in stunting & $10 \%$ \\
Community-based preventive and promotional services & $3 \%$ \\
Changes in breastfeeding practice & $3 \%$ \\
Not reported & $11 \%$ \\
Total & $100 \%$ \\
\hline
\end{tabular}

ORS=oral rehydration solution. Hib=Haemophilus influenzae type b. ITN=insecticide-treated bednet.

Table: Relative contribution of each strategy to reduction of under- 5 mortality rate (U5MR ${ }^{1,3}$

access to curative services. Page and colleagues $^{2}$ report that $40 \%$ of care for children had been provided by CHWs in 2009. In Niger, there is an even more peripheral level of relais communautaire. Further evidence of the role lay health workers can have in resource-constrained settings, especially the respective roles of the two levels of $\mathrm{CHWs}$, should be sought.

Mass campaigns, most notably distribution of insecticide-treated bednets, account for $45 \%$ of U5MR decline (table). ${ }^{1,3}$ The uncertain sustainability and unintended consequences ${ }^{4}$ of these repeated campaigns on health systems should be documented. Nutrition programmes have contributed $19 \%$ to the reduction of U5MR (table). That reduction of stunting could have contributed $10 \%$ to the reduction in U5MR is difficult to understand, considering that overall stunting rates did not decline and that food security did not improve.

Better understanding of the mechanisms that enabled these three strategies to achieve the estimated effect in this context could generate important lessons for future reforms in Niger and globally. Several frameworks have been developed recently to help deepen our understanding of health systems that could guide additional in-depth case studies. ${ }^{5}$

We are part of the external evaluation of the Catalytic Initiative to Save a Million Lives, which is funded by the Canadian International Development Agency being Niger.

*David Hercot, Tanya Doherty, Charles Hongoro, Wim Van Damme, David Sanders

\section{dhercot@gmail.com}

Public Health Department, Institute of Tropical Medicine, 2000 Antwerp, Belgium (DH, WVD); Health Systems Research Unit, Medical Research Council, Parow, Cape Town, South Africa (TD, CH); Tshwane University of Technology, Pretoria, South Africa $(\mathrm{CH})$; and School of Public Health, University of the Western Cape, Bellville, Cape Town, South Africa (TD, DS)

1 Amouzou A, Habi O, Bensaid K, for the Niger Countdown Case Study Working Group. Reduction in child mortality in Niger: a Countdown to 2015 country case study. Lancet 2012; 380: 1169-78.

2 Page AL, Hustache S, Luquero FJ, Djibo A, Manzo ML, Grais RF. Health care seeking behavior for diarrhea in children under 5 in rural Niger: results of a cross-sectional survey. BMC Public Health 2011; 11: 389.

3 Chopra M, Sharkey A, Dalmiya N, Anthony D, Binkin N. Strategies to improve health coverage and narrow the equity gap in child survival, health, and nutrition. Lancet 2012; 380: $1331-40$ through UNICEF in six African countries, one of them
4 Doherty T, Chopra M, Tomlinson M, Oliphant N, Nsibande D, Mason J. Moving from vertical to integrated child health programmes: experiences from a multicountry assessment of the Child Health Days approach in Africa. Trop Med Int Health 2010; 15: 296-305.

5 Van Olmen J, Criel B, Bhojani U, et al. The health system dynamics framework: the introduction of an analytical model for health system analysis and its application to two case-studies. Health Culture Society 2012; 2: 1-21.

Agbessi Amouzou and colleagues ${ }^{1}$ provide a vital contribution in analysing the remarkable progress in reduction of child mortality in Niger. However, best available data have not been used in reporting the history of campaign approaches and coverage of vitamin A supplementation (VAS). The contribution of high, twice-yearly VAS coverage-one of Niger's signature child survival achievements-to this dramatic reduction in child deaths is therefore likely to be a major underestimate.

Niger started campaigns in 1996, combining VAS with vaccination, and continued once-per-year mass VAS distribution until 1999. It was one of the first countries in sub-Saharan Africa to ensure twice-yearly VAS coverage (starting in June, 1999). ${ }^{2,3}$ Amouzou and colleagues focus only on campaign approaches starting in 2004, stating that there were two mass campaigns per year for VAS since 2000.

VAS coverage, presented in Amouzou and colleagues' appendix, is significantly lower than that from other validated sources. Figure 7 shows coverage ranging from about $60 \%$ to 80\% during 2005-09 (with no data between 1998 and 2005). In fact coverage has been maintained at more than $80 \%$ since June, $1999.2,3$

Finally, in their use of the Lives Saved Tool (LiST), Amouzou and colleagues have only factored in VAS's effect on reduction of diarrhoeaspecific mortality and use of VAS in treatment of measles, whereas several meta-analyses have shown VAS as producing a $22-25 \%$ reduction in all-cause mortality for children aged 6-59 months in settings such as 